\title{
Numerical investigation of vortex shedding and vortex-induced vibration for flexible riser models
}

\author{
Zheng-Shou Chen ${ }^{1,3}$ and Wu-Joan Kim ${ }^{2}$ \\ ${ }^{1}$ School of Naval Architecture and Civil Engineering, Zhejiang Ocean University, Zhoushan, China \\ ${ }^{2}$ Department of Ocean Engineering, Mokpo National University, Mokpo, Korea \\ ${ }^{3}$ Regional Innovation Center for Southwestern Midsized Shipbuilding Industry, Mokpo, Korea
}

\begin{abstract}
The numerical study about the vortex-induced vibration and vortex shedding in the wake has been presented. Prior to the numerical simulation of flexible riser systems concerning engineering conditions, efficiency validating of the proposed FSI solution method have been performed. The comparison between numerical simulation and published experimental data shows that the CFD method designed for FSI solution could give acceptable result for the VIV prediction of flexible riser/pipe system. As meaningful study on VIV and vortex shedding mode with the focus on flexible riser model systems, two kinds of typical simulation cases have been carried out. One was related to the simulation of vortex visualization in the wake for a riser model subject to forced oscillation, and another was related to the simulation of fluid-structure interaction between the pipes of coupled multi-assembled riser system. The result from forced oscillation simulation shows that the vortexinduced vibration with high response frequency but small instantaneous vibration amplitude contributes to vortex conformation as much as the forced oscillation with large normalized amplitude does, when the frequency of forced oscillation was relatively high. In the multi-assembled riser systems, it has been found that the external current velocity and the distance between two pipes are the critical factors to determine the vibration state and the steady vibration state emerging in quad-pipe system may be destroyed more easily than dual-pipe system.
\end{abstract}

KEY WORDS: Vortex-induced vibration (VIV); Vortex shedding; Forced motion; Multi-assembled pipe; FSI.

\section{INTRODUCTION}

Flow around a fixed or oscillating cylinder has received lots of attention in the past few decades. With the massive use of flexible risers, pipelines, mooring lines and underwater cables in deepwater oil production and offshore wind energy utilization industries, the demand to focus on the research of vortex-induced vibration (VIV) and vortex shedding in the wake has been greatly enhanced. Those flexible risers/pipes are readily subjected to shear and oscillatory flows due to currents and waves with high degree of complexity, and with intensity and direction changes according to water depth. Among those ocean destructive factors, vortex-induced vibration plays a leading role in determining the life span of marine risers. In the so-called "lock-in" or "synchronization" state, the resonance with amplified vibration amplitude occurs, leading to significant fatigue damage to marine riser systems.

Complicated as real VIV problems are, due to the limitation of calculation ability and some shortages of CFD software package itself, most numerical results relied on 2D vortex simulation and then integrated the hydrodynamic force

Corresponding author: Wu-Joan Kim

E-mail:kimwujoan@mokpo.ac.kr to 3D riser structure (Yamamoto and Meneghini, 2004). Some attempts to simulate VIV with very large aspect ratio are indeed 2D simulations (Holmes and Owen, 2006). Kim and Rheem (2009) measured the cross flow response of a cylinder under local shear flow.

With risers presenting high aspect ratio and complex vortex pattern around them, a well-performed 3D simulation method which is specially designed for FSI solution should be looked for. Among those pioneering endeavors, Menter et al. (2006) has successfully solved two FSI simulations by ANSYS multi-physics software. It has preliminarily verified the capability of ANSYS MFX solver to deal with coupled fluid-structure problem related to flexible pipe/riser model. In this paper, the FSI problems concerning riser models subject to engineering conditions have been solved by means of MFX solution approach too. Prior to applying the CFD method to deal VIV problem arbitrarily, the efficiency validation of MFX solver had been performed first.

\section{NUMERICAL SOLUTION}

\section{FSI strategy of ANSYS}

ANSYS MFX solver has been adopted to solve the twoway FSI problems related to VIV of flexible riser systems. 
The well-designed ANSYS FSI solution strategy provides tightly integrated flow and structure physics, offering designers and analysts a most flexible and advanced coupled fluid-structure physics analysis tool. In addition, the implicit coupling iteration ensures the consistency between fluid and structure solution fields at the end of each multi-field step, leading to improved solution stability (ANSYS, 2007).

\section{Turbulence model}

Generally, the discrepancy between experiments and numerical simulations attributes much to the disability of turbulence model and the time-space resolution of the grid used in numerical simulation. Due to the Reynolds number of all the simulation cases in this paper not high, the LES model which is proposed for cases with large scale flapping of shear layers or vortex shedding has been adopted.

\section{EFFICIENCY VALIDATION OF FSI SOLUTION}

The simulation for numerical efficiency validation was related to the published VIV experiment concerning a naked brass pipe under uniform and sheared currents, carried out by MARINETEK. The riser model was made of a brass pipe with outer diameter of $20 \mathrm{~mm}$ and wall thickness of $0.45 \mathrm{~mm}$. The effective length of the riser model was $9.63 \mathrm{~m}$. The tension imposed on the two ends was $817 N$. More information should refer to the experimental description document and some accomplished numerical simulations related to this VIV experiment (Lehn, 2003; Huang and Chen, 2009).

Two kinds of simulations related to uniform and sheared currents, which the brass riser system was subject to respectively, have been performed. It is worth noting that the sheared current speed has a linear profile with $V_{\text {min }}$ (corresponding to the position of "0" in Figs. 1 2) at bottom and $V_{\max } \quad$ (corresponding to the position of "1" in Figs. 1 2) at top, where $V_{\min } / V_{\max }=0.14$. Two cases, with $V_{\max }=$ $0.20 \mathrm{~m} / \mathrm{s}, \quad 0.42 \mathrm{~m} / \mathrm{s}$, were selected for representative comparison between simulation and experiment in the case of sheared current condition. Another case with uniform current velocity of $0.84 \mathrm{~m} / \mathrm{s}$ was also presented, in order to validate the efficiency of ANSYS multi-field software to deal with case with high reduced velocity.

The RMS A/D (where A is the normalized instantaneous vibration amplitude and $\mathrm{D}$ is the riser diameter) patterns of cross-flow response along the riser span are shown in Figs. $1 \sim 3$, corresponding to sheared current of $0.20 \mathrm{~m} / \mathrm{s}$ and $0.42 \mathrm{~m} / \mathrm{s}$ and uniform current of $V=0.84 \mathrm{~m} / \mathrm{s}$, respectively. Generally speaking, the comparison result is encouraging. In the case of sheared current of $0.20 \mathrm{~m} / \mathrm{s}$, shown in Fig. 1, the CFD simulation result agreed well with the observed data from model test case of No. 1201 (Lehn, 2003). The disagreement became slightly obvious when the sheared current rose to $0.42 \mathrm{~m} / \mathrm{s}$. The magnitude and position estimations of the cross-flow response along the riser span deviated a little from the result of mode test case of No. 1205, as shown in Fig. 2.
However, the apparent waveform of the second mode consisted with the observation from VIV experiment. Considerable disagreement has been found in the case of uniform current velocity equal to $0.84 \mathrm{~m} / \mathrm{s}$, i.e. VIV experiment case of No. 1108. As it is shown in Fig. 3, the CFD method predicted that the 4th mode was the apparent dominant mode, while the experimental data indicated that both the $3^{\text {rd }}$ and $4^{\text {th }}$ modes had been excited simultaneously. The disagreement between numerical and experimental observations attributes much to the underestimation of multimodal vibration state by numerical method. It is consistent with the conclusion drawn by the authors of this paper that the emergence of subordinate vibration models even with tiny vibration power would lead to considerable energy transition from one vibration plane to another (Chen and Kim, 2010).

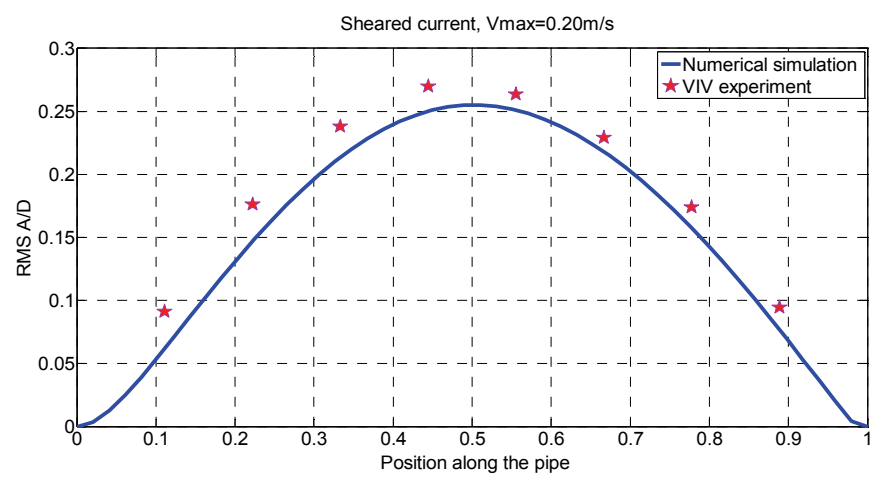

Fig. 1 RMS $A / D$ pattern of cross-flow response along the brass riser span, in the case of sheared current $V_{\max }=0.20 \mathrm{~m} / \mathrm{s}$.

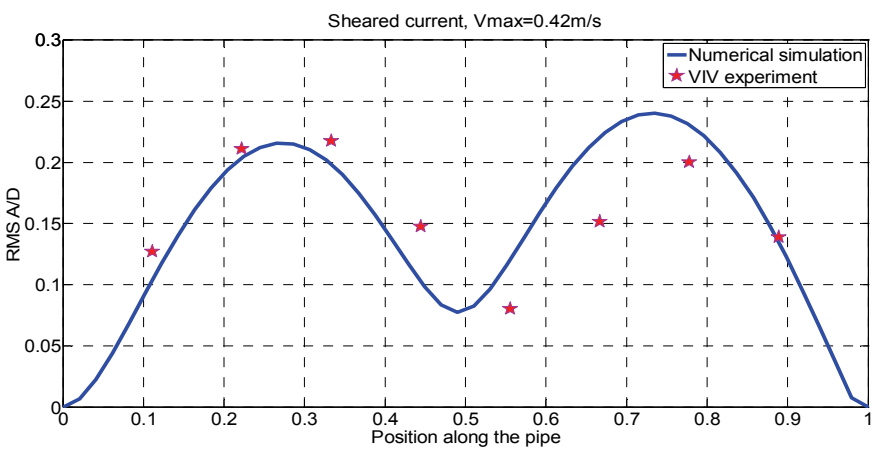

Fig. 2 RMS $A / D$ pattern of cross-flow response along the brass riser span, in the case of sheared current $V_{\max }=0.42 \mathrm{~m} / \mathrm{s}$.

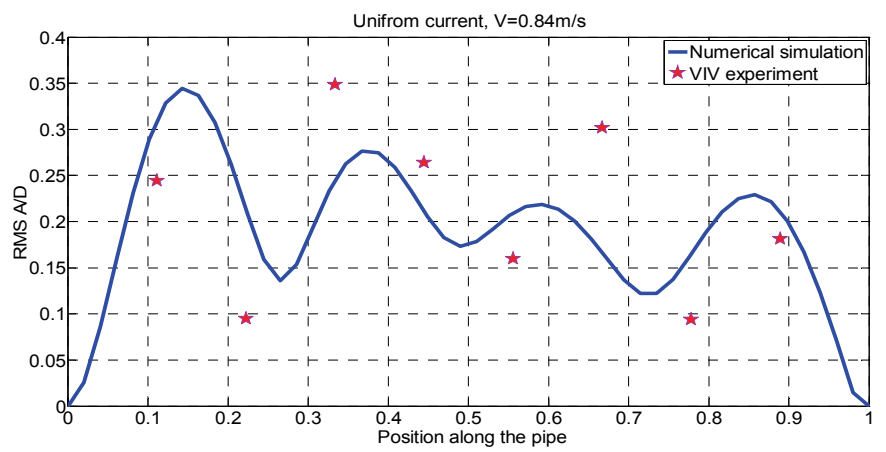

Fig. 3 RMS $A / D$ pattern of cross-flow response along the brass riser span, in the case of uniform current $V=0.84 \mathrm{~m} / \mathrm{s}$. 
Huang et al. (2009) has similar conclusions with those obtained in this paper when carried out similar numerical simulation related to this VIV experiment, that is, the disagreement being popular while current velocity becoming considerably large. Insufficient as it was, the CFD simulation still provided approximate riser vibration response for further hydrodynamics analysis. Another numerical simulation concerning the efficiency validation of ANSYS MFX solver for flexible riser model was carried out by the authors of this paper in their previous work (Chen and Kim, 2008). In that case, the obtained simulation result has a good agreement with the observation from the related VIV experiment performed by KORDI.

In a word, the estimation from the above two simulations shows comparatively good agreement with VIV experiment from the point of view of engineering accuracy requirement. The disagreement (in magnitude, position and dominant mode estimation) between simulated and observed experiment data partially results from the numerical simplification which had been done to speed up the simulation convergence, and mainly from the unpredictable effect of the rotation rig system. In fact, when the external current velocity rises, even some slightly influencing factors can easily become enlarged or dominant. Those phenomena are more distraught in model test than numerical simulation in which some assumption and simplification have usually been adopted. By this FSI solution method, the numerical simulation related to forced vibration and multi-pipe system had been carried out.

\section{RISER MODEL SUBJECT TO FORCED MOTION}

\section{Problem descriptions}

The model test upon which this simulation case based was performed at KORDI (Choi and Hong, 2000). The riser model was made of a $3.4 m$ long PVC pipe coated by rubber with the external diameter of $53.8 \mathrm{~mm}$. The pipe upper end was forced to translate in a sinusoidal trajectory with definite amplitude, and another (namely, lower end) was drawn by an object weight $1 \mathrm{~kg}$, which was free to vibrate in all directions. The frequency of forced sinusoidal oscillation varied from $0.2 \mathrm{~Hz}$ to $2 \mathrm{~Hz}$.

\section{Vortex visualization in the wake}

The objective of this simulation was to provide VIV experiment some supplementary information of wake shedding and give reasonable interpretation to structural oscillation mode in the form of dynamic analysis between fluid and structure.

Four cases corresponding to the forced frequency of $0.61 \mathrm{~Hz}, 1,06 \mathrm{~Hz}, 1.43 \mathrm{~Hz}, 1.60 \mathrm{~Hz}$ (denoted as Cases 2, 3, 5, and 6, respectively in Fig. 4), have been selected to show the effect of forced vibration and the subsequently vortex induced vibration on the flexible riser system.
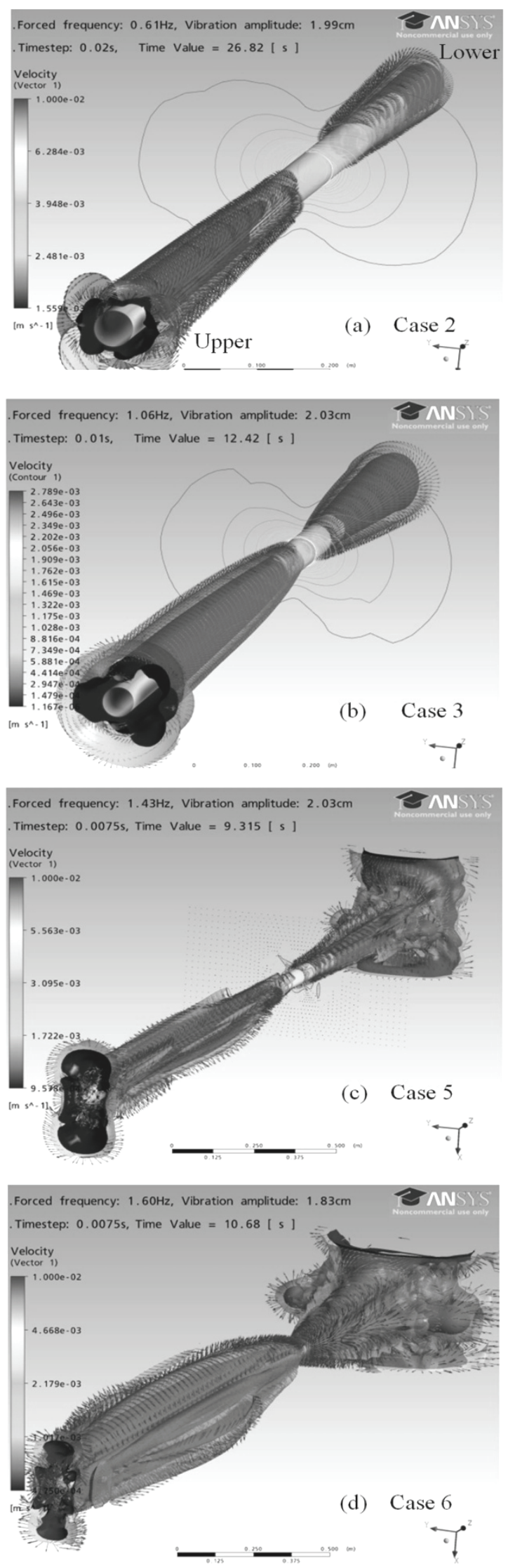

Fig. 4 The isovelocity surface visualization around the oscillating PVC pipe at different frequencies. 
The blue opaque envelope represents the isovelocity surface of $\mathrm{V}=0.02 \mathrm{~m} / \mathrm{s}$, and green semi-transparent envelope denotes the isovelocity surface of $\mathrm{V}=0.01 \mathrm{~m} / \mathrm{s}$. From the comparison analysis between those four cases, it was observed that the instantaneous isovelocity surface and visualization of velocity vector varied with the instantaneous deformation of riser system. However, the relative position of isovelocity surface to the lengthwise deformed surface was comparatively steady. As a result, it became a measurement of vibration intensity, just as the instantaneous normalized amplitude $(A / D)$ does in the analysis of vibration intensity from the point of view of structural dynamics.

Since the riser model was not long enough, only the second intrinsic vibration model has been observed in those four cases. As it is shown in Fig. 4, the vibration process with the second mode being arisen was demonstrated by the visualization of dumbbell-like wake pattern around riser span in the fluid domain. In the case of low frequency with small excited instantaneous amplitude, e.g. Cases 2 and 3, isovelocity surfaces were relatively steady, being approximately of a pair of circular cones, demonstrated in the subplots (a) and (b) of Fig. 4. The isovelocity surface pattern changed a lot, when the oscillation frequency increased. The conical isovelocity surface appeared in the case of low vibration frequency turned entirely into distorted envelope in Cases 5 and 6 . This was mainly due to the promptly increased structural vibration speed which was characterized by high oscillation frequency and considerable vibration amplitude. Another reason should attribute to the presence of threedimensional flow condition, known as "end effect", which is commonly considerable when flow velocity is high and the aspect ratio (L/D) is not large (Michael, 1994). From the illustration comparison between the subplots in Fig. 4, one may find that the intensity of "end effect" became evitable and extended to the field far from both ends in the case with large vibration frequency, i.e. Cases 5 and 6 . It is worth nothing that the emergence of "end effect" resulted partly from the occurrence of internal progressive flow induced by the coupled effect of the in-plan forced oscillation and out-ofplane vortex-induced vibration. The "end effect" at the riser lower end was extremely magnified, shown in subplots (c) and (d) of Fig. 4, for the outflow of vibration-induced internal progressive flow became considerable when oscillation frequency rose.

Another observation was that the magnitude variation of velocity vector in Y-axis direction (namely, in-plane) which was located along about the upper $2 / 3$ riser length was more serious than that observed in X-axis direction (namely, outof-plane). It is equivalent to the visualization that the isovelocity surfaces of $\mathrm{V}=0.01 \mathrm{~m} / \mathrm{s}$ was thin in in-plane and became thick in out-of-plane vibration direction. As a rule, in those four cases, the fast the forced oscillation was, the large the intensity difference became. It means that the magnitude of force oscillation frequency determines the intensity of inplane oscillation distinctly.

Although the structural deformation of out-of-plane vibration has several orders of magnitude less than that of inplane, the velocity magnitude in the two directions (on the same section of pipe span) was almost equivalent along the lower $1 / 3$ riser length. In the same way, the velocity magnitude was also comparable along the upper $2 / 3$ riser length. The Y-axis velocity component mainly resulted from the large displacement and deformation of in-plane induced by forced oscillation, while the $\mathrm{X}$-axis velocity component was mainly due to the vortex-induced vibration in out-ofplane. Since the magnitude of velocity vector induced by VIV has the same order of magnitude with the former, it should not be negligible. Hereon, one may draw a conclusion that the out-of-plane vortex-induced vibration with relatively small normalized instantaneous amplitude but high vibration mode contributes to the vortex wake conformation as much as the forced oscillation did which often follows with large normalized amplitude.

\section{FLEXIBLE MULTI-ASSEMBLED PIPE SYSTEMS}

\section{Problem descriptions}

The main purpose of this section is to apply the FSI solution to analyze the VIV phenomena of multi-assembled risers commonly used in offshore oil production. The riser model was made of PVC pipes with an outer diameter of $50 \mathrm{~mm}$ and a wall thickness of $2.5 \mathrm{~mm}$. The basic configuration of the numerical simulation and boundary condition setting were shown in Fig. 5. In this paper, riser systems with two (in tandem) and four (equidistance in tandem and left and right sides) pipe assemblies were taken into account, focusing on the interaction between front and rear pipes.

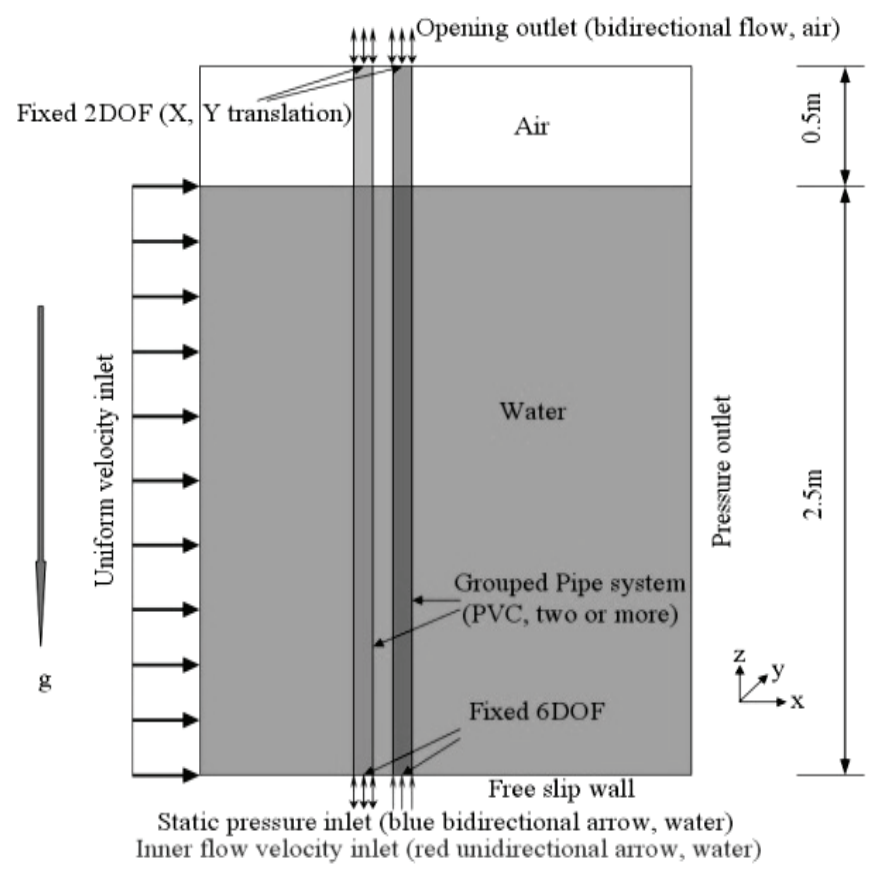

Fig. 5 The configuration of the multi-pipe system and the boundary conditions in numerical simulation. 


\section{Analysis of dual-pipe system}

Two kinds of semi-submarine dual-riser systems has been taken into account, with the central axis distance $d$ between pipes of $2 D$ and $3 D$ respectively, where $D$ represents the pipe outer diameter. Six cases, corresponding to different combinations of external current velocity $V_{\text {ext }}(0.2 \mathrm{~m} / \mathrm{s}, 0.4 \mathrm{~m} / \mathrm{s}$ and $0.6 \mathrm{~m} / \mathrm{s}$ respectively) and central distance $d(2 D, 3 D)$, were performed to analyze the VIV amplification happening in the dual-riser systems. Another six cases aiming at the comprehension of interaction between internal progressive flow $V_{\text {in }}$ and external current $V_{\text {ext }}$ were also accomplished, by setting $V_{\text {in }}$ and $V_{\text {ext }}$ to be of $0.2 \mathrm{~m} / \mathrm{s}, 0.4 \mathrm{~m} / \mathrm{s}$ and $0.6 \mathrm{~m} / \mathrm{s}$, respectively, where $V_{i n}=V_{\text {ext }}$.

When the two pipes in tandem was close enough, e.g. $2 D$ in this paper, the destruction, retardation and reconstruction of vortices affected the vortex conformation in the wake distinctly. Here, the magnitude of velocity component towards the tail end of front pipe (namely, backflow) has been enlarged obviously, leading to prompt decline of drag efficient. As a result, the magnitude decline of drag coefficient was considerably large, comparing to the variation of lift force. In most cases of $d=2 D$, neither the vibration amplitude nor the oscillation frequency was constant, as the shear layers and vortex structures had never been sufficiently

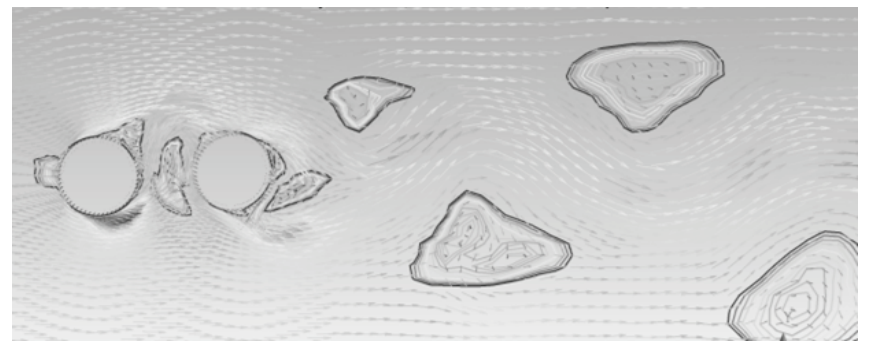

Fig. 6 Vortex shedding pattern for the case of $d=2 D$, $V_{\text {ext }}=0.4 \mathrm{~m} / \mathrm{s}$, at position $Z=1.8 \mathrm{~m}$.

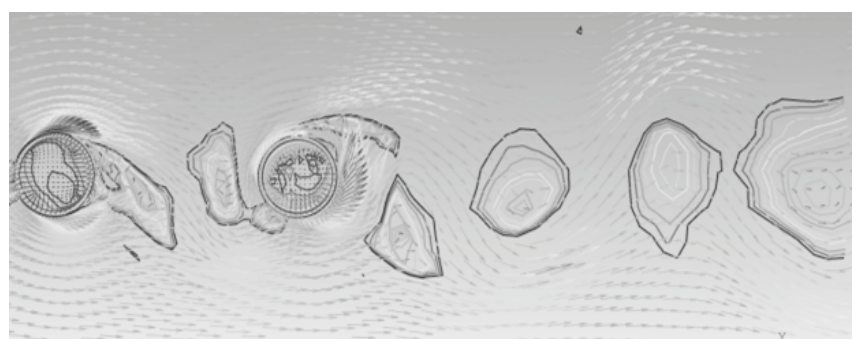

Fig. 8 Vortex shedding pattern for the case of $d=3 D$, $V_{\text {ext }}=0.4 \mathrm{~m} / \mathrm{s}$, at position $Z=1.8 \mathrm{~m}$.

\section{Analysis of quad-pipe system}

Furthermore, VIV numerical simulation for quad-pipe configuration (center-to-center distance of 2D in-line and 2D cross-flow) has been performed, with $V_{\text {ext }}=0.2 \mathrm{~m} / \mathrm{s}, 0.4 \mathrm{~m} / \mathrm{s}$ established. In the case of $d=2 D, V_{e x t}=0.6 \mathrm{~m} / \mathrm{s}$ the $\mathrm{P}+\mathrm{S}$ vortex shedding pattern (a pair of vortices and single vortex each side) has been observed in the vortex wake around the pipe mid-span, as shown in Fig. 7. It resulted from the fact that the coupling became distinct when the pipes in tandem were closer and the reduced velocity was larger. In this case, the existence of rear pipe played an important role in reconstructing the vortex shedding wake behind the front pipe. It finally resulted in considerable VIV amplification in cross-flow direction and considerable velocity fluctuation in in-line direction from cycle to cycle. Since the length of flexible pipe was not long enough to arise considerable vibration amplitude, the $2 \mathrm{P}$ shedding mode (a pair of vortices both sides) has not been found in all of the cases. The $2 \mathrm{~S}$ shedding mode (single vortex both sides) was prevailing in most simulation cases, shown in Figs. 6, 8 and 9.

Although the existence of internal flow effectively influenced the cross-flow VIV and dominant frequency in the case of $V_{\text {ext }}=V_{\text {in }}=0.4 \mathrm{~m} / \mathrm{s}$, the external current velocity and the distance between two pipes were still the critical factors to determine the vibration state of the dual-pipe riser system. Contrasting to those conclusions drawn on long riser model (Chen and Kim, 2010), the effect of internal progressive flow on VIV of the rear pipe was restricted, due to the small aspect ratio $L / D$ of the pipe adopted in the present study.

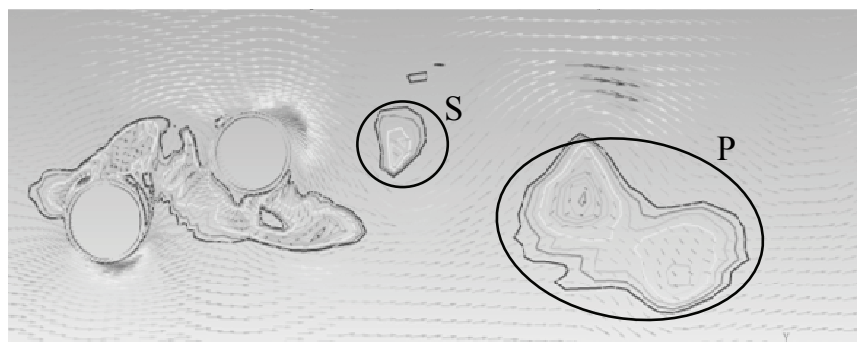

Fig. 7 Vortex shedding pattern for the case of $d=2 D$, $V_{\text {ext }}=0.6 \mathrm{~m} / \mathrm{s}$, at position $Z=1.8 \mathrm{~m}(\mathrm{P}+\mathrm{S}$ type vortex model appears with enlarged amplitude).

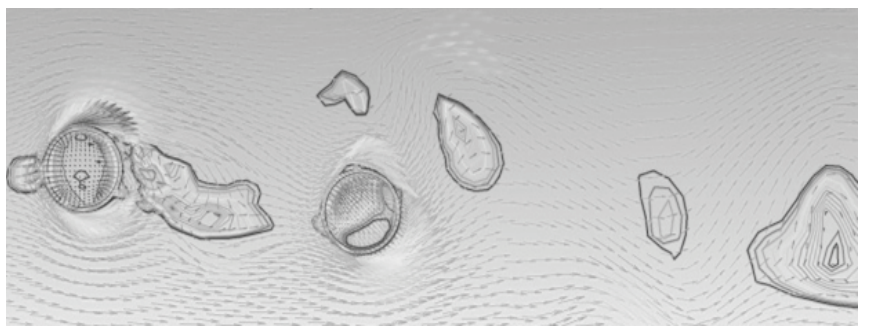

Fig. 9 Vortex shedding pattern for the case of $d=3 D$, $V_{\text {ext }}=0.6 \mathrm{~m} / \mathrm{s}$, at position $Z=1.8 \mathrm{~m}$.

and without internal flow being considered. The vortex shedding pattern and normalized time history corresponding to external current velocity $V_{\text {ext }}$ of $0.40 \mathrm{~m} / \mathrm{s}$ and $0.20 \mathrm{~m} / \mathrm{s}$ are shown in Figs. 10 and 11 (at the position of the $Z=1.8 \mathrm{~m}$ ), respectively. 

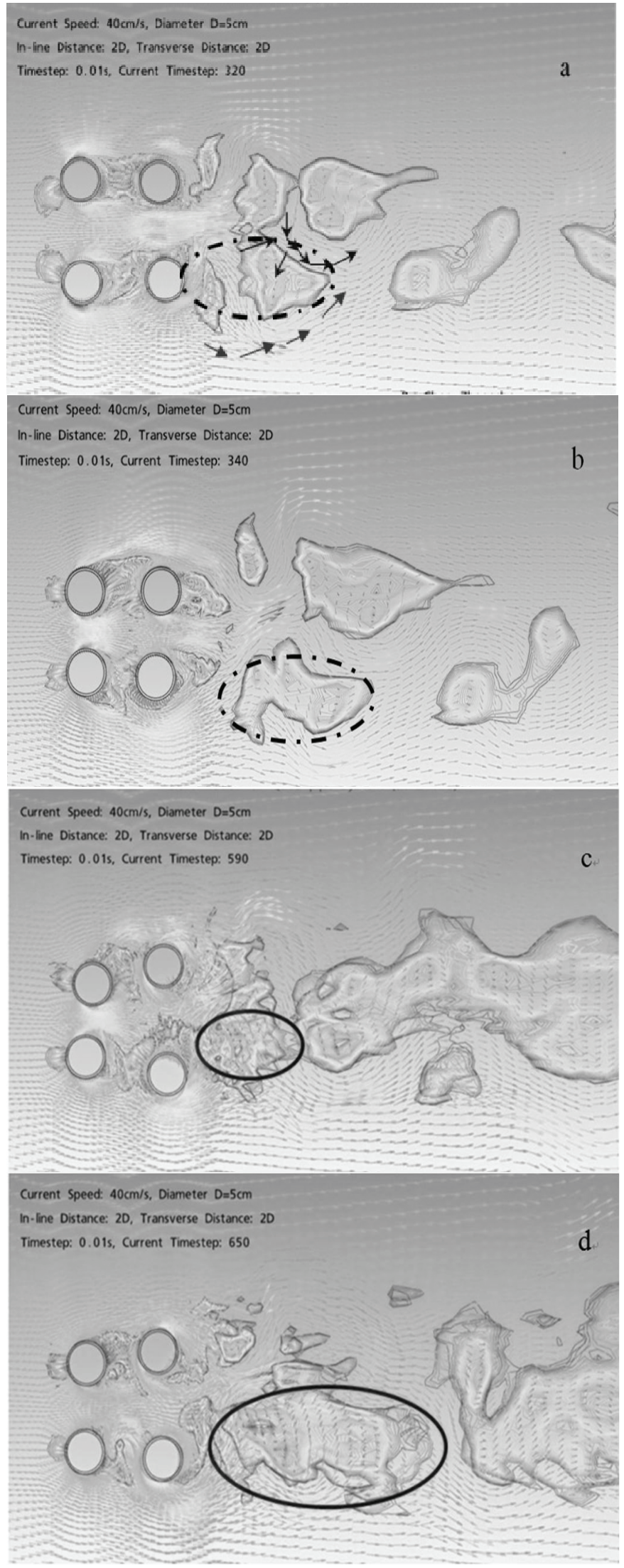

Fig. 10 Vortex visualization for the case of quad-pipe system confined by 2D in-line and 2D cross-flow with $V_{\text {ext }}=0.4 \mathrm{~m} / \mathrm{s}$, at position $Z=1.8 m$.
As that is shown Fig. 10, the vortex shedding patterns were more complex than those observed in dual-pipe systems, when $V_{e x t}=0.40 \mathrm{~m} / \mathrm{s}$. Among those unique visualizations, it was found that there were some fields where the vortex regions were enlarged, shown in subplots (c) and (d) of Fig. 10. It attributed to the fact that the rolling directions of two neighbor S-type vortices which came from two adjacent rear pipes were co-rotating. However, there were some regions where the vortex conformation was shrunk or even disappeared when vortex directions of adjacent vortices were contra-rotating, demonstrated in subplots (a) and (b) of Fig. 10 .

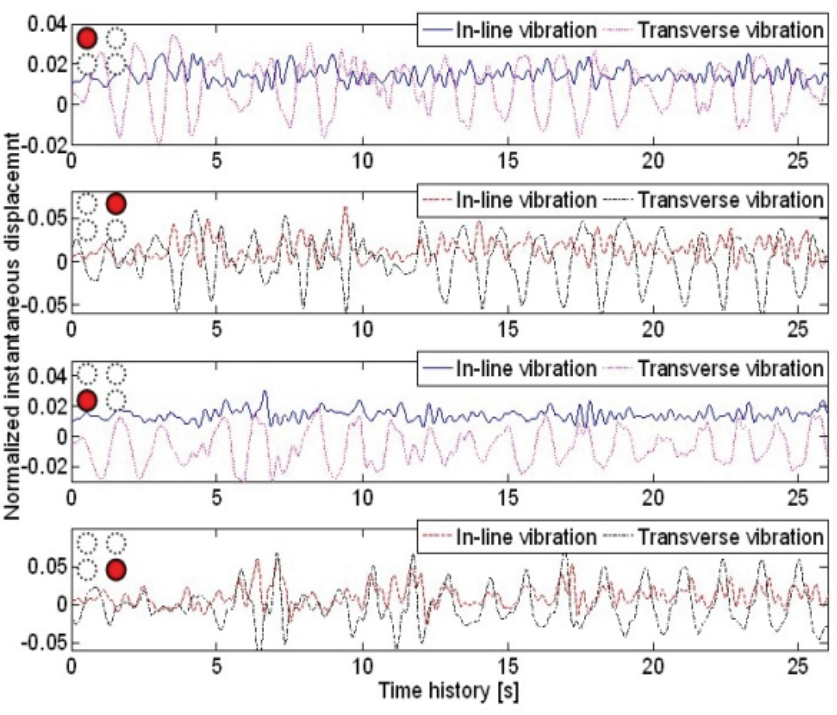

Fig. 11 The normalized time history the quad-riser system with $V_{e x t}=0.2 \mathrm{~m} / \mathrm{s}$, at position $Z=1.8 m$ (the red point shows the demonstrating riser position in the system).

In the case of $V_{\text {ext }}=0.20 \mathrm{~m} / \mathrm{s}$, shown in Fig. 11, both inline and cross-flow vortex-induced vibrations were similar with those observed in single-pipe system, for the reduced velocity was not large enough to excite vortex shedding mode with high frequency. The instantaneous oscillation amplitude of in-line vibration was an order of magnitude lower than that observed in cross-flow vibration. Although the backflow had been enhanced due to the existence of downstream pipe, it was found that it had little influence on the drag coefficient of front pipes.

Similar with those found in the case of dual-pipe system, the vortex shedding conformation was controlled by the existence of the rear pipes at low external flow velocity. Nevertheless, the periodic VIV state would be destroyed more quickly than dual-pipe system when the reduced velocity became higher, e.g. $V_{\text {ext }}=0.40 \mathrm{~m} / \mathrm{s}$ in this paper. In addition, due to the mutual restriction from adjacent pipes in cross-flow and in-line simultaneously, both the RMS values of in-line and cross-flow VIVs were larger than those observed in dual-pipe system. 


\section{CONCLUDING REMARKS}

A series of VIV-related research works with the focus on flexible riser models subject to two kinds of engineering conditions have been performed in this paper. The main conclusions from above study have been drawn as follows.

Efficiency validation of ANSYS MFX solver designed for FSI solution has been carried out based on published VIV experiment. It has been proved that that the numerical simulation shows good agreement with VIV experiment when the maximum current velocity is relatively low. The increasing disagreement related to the case of high reduced velocity attributes much to the uncertainty and complexity of the actual rotation rig system and discrepancy of different assumption adopted by numerical and experiment methods.

Numerical simulation accomplished in this paper reveals some new findings concerning complex riser systems. The result from forced oscillation simulation shows the VIV with high frequency contributes to vortex conformation as much as the forced oscillation with large normalized amplitude does. In the multi-assembled pipe systems, it has been found that the external current velocity and the distance between two pipes are the critical factors to determine the vibration state and the steady vibration state in quad-pipe system may be destroyed more easily than dual-pipe system. Totally, the important findings from the numerical simulation contribute much to a reasonable interpretation of the coupling mechanism between VIV and vortex wake.

\section{ACKNOWLEDGEMENTS}

This research work was financially supported by Korea Research Foundation Grant funded by the Korean Government (MOEHRD, Basic Research Promotion Fund) (KRF-2008-D00556) and Korean Regional Innovation Center (RIC) for Southwestern Midsized Shipbuilding Industry project in the year of 2010, Mokpo National University.

\section{REFERENCES}

ANSYS, 2007. ANSYS CFX-Solver Modeling Guide (V11 SP1), ANSYS Co.

Chen, Z.S. and Kim, W.J., 2008. Numerical simulation of a large-scale riser with vortex-induced vibration. Proceedings of the Eighth ISOPE Pacific/Asia Offshore Mechanics Symposium, Bangkok, Thailand. pp.121-128.

Chen, Z.S. and Kim, W.J., 2010. Effect of internal flow on fluid-structure interaction of marine riser model subject to sheared current. submitted to Journal of Fluids and Structures.

Choi, Y.L. and Hong, S., 2000. Study about structural deformation estimation about flexible pipe in the engineering basin model test. Korea Ocean Research \& Development Institute (KORDI), Research report.

Holmes, S. and Owen, H., 2006. Simulation of Riser VIV Using Fully Three Dimensional CFD Simulations. OMAE, Hamburg, Germany, 4-9 June.

Huang, K. and Chen, H.C., Chen, C.R., 2009. Vertical Riser VIV Simulation in Sheared Current. ISOPE, Osaka, Japan. 21-26 June, pp. 1369-1376.

Lehn, E., 2003. VIV suppression tests on high L/D flexible cylinders (main report). ExxonMobil upstream research company.

Menter, F. and Sharkey, P., 2006. Overview of Fluidstructure coupling in ANSYS-CFX. 25th International Conference on Offshore Mechanics and Arctic Engineering, Hamburg, Germany.

Michael, S. P., 1994. Vortex induced vibration parameters: critical review. OMAE Offshore Technology, USA.

Yamamoto, C.T. Meneghini, J.R. Saltara F. Fregonesi, R.A., and Ferrari, J.A. Jr, 2004. Numerical simulations of vortex-induced vibration on flexible cylinders. Journal of Fluids and Structures, 19(4), pp. 467-489.

Kim Y.C. and Rheem C.K., 2009, Cross flow response of a cylindrical structure under local shear flow, International Journal of Naval Architecture and Ocean Engineering, 1(2), pp. 101 107. 